are inadequate to control pain. Our aim was to systematically review all studies that report pharmacological and non-pharmacological interventions for refractory angina, and discuss palliative interventions appropriate for hospice and community-based care.

Methods We performed a literature search of six databases and a search of available grey literature. Studies relating to first- and second- line pharmacological treatments were excluded, as were interventions that had undergone systematic review within the last three years.

Results 4475 studies were screened, 37 studies were included in our analysis. Interventions were cardiac shockwave therapy (twenty two), transcutaneous electrical nerve stimulation (TENS) (four), perhexiline (two), optimal medical therapy (two), multi-disciplinary care programmes (two), psychotherapy (two), cardiac rehabilitation (one), morphine (one) and intranasal alfentanil (one). The majority of studies reported positive results, with improvements in pain, angina class, exercise tolerance and quality of life. Very few adverse effects were reported across all studies but quality assessment was varied and risk of bias was generally high.

Discussion There is a significant body of literature regarding interventions for refractory angina that is over-looked in current clinical practice. While the quality of these studies varies, improvements have been reported in symptom control and quality of life with few adverse effects. There is a need for further research into these interventions which could be useful within the contexts of cardiology and palliative care.

\section{P-123 CALCIPHYLAXIS AND THE CHALLENGES OF ADVANCE CARE PLANNING IN END STAGE RENAL DISEASE}

Natasha Palipane, Srirupa Gupta. Farleigh Hospice, Chelmsford, UK

10.1136/bmjspcare-2019-HUKNC.146

Background Calciphylaxis is a rare and poorly understood complication of end stage renal disease (ESRD) that causes a complex pain difficult to manage in the acute hospital and hospice environment. Its management is further confounded by the symptom burden of ESRD and the challenges of facing the extremely poor prognosis associated with this condition.

Aim We aim to share our experiences in treating this condition using a holistic, cross-specialty approach. We would also like to suggest our guidance in managing the physical and psychosocial symptoms of calciphylaxis whilst exploring advance care planning (ACP) in ESRD in a short time frame.

Method We present a case of a patient newly diagnosed with calciphylaxis on a background of ESRD. The patient was managed by the hospice palliative care team with input from the renal physicians in both the acute hospital and hospice environment, spanning a period of two months from acute presentation to death. The case illustrates the impacts of various methods used in treating the patient's symptoms. There is also a review of the evidence and guidelines for the use of opioids in ESRD as well as the guidance on ACP in ESRD.

Results We found that a cross-specialty approach is key to managing the symptoms of calciphylaxis and ESRD even when dialysis is discontinued. A variety of analgesics and formulations were required in treating the associated ischaemic pain. In utilising the multidisciplinary team, a comfortable death was achieved whilst placing the patient's choices at the centre of their care.

Conclusions In treating the complex symptom burden and unusual presentations of ESRD, a close working relationship between the palliative medicine and renal team is essential. ACP conversations are desired by these patients but can be infrequent in reality and should be instigated earlier and repeatedly throughout their patient journey.

\section{P-124 EVOLUTION AND REVOLUTION IN MANAGING HOSPICE PATIENTS' BLOOD GLUCOSE LEVELS}

Sarah Bell. Garden House Hospice Care, Letchworth Garden City, UK

10.1136/bmjspcare-2019-HUKNC. 147

Background On outpatient review, a recent in-patient initiated on steroids was newly diabetic. Given the minimal hospice guidance on blood glucose management, a quality improvement programme was initiated.

Aim Evolution and revolution in care to provide high standards of blood glucose management in patients taking steroids, with diabetes or nearing end of life.

Method Process over four years, informed by three audit cycles.

Firstly, development of hospice standards for blood glucose management in patients with diabetes or on steroids, based on Diabetes UK End of Life Diabetes Care Recommendations (3rd ed., 2018), which was adopted as hospice policy. Overall, standards were to ensure:

- Recording of blood glucose levels and documentation of clear, appropriate management plans for diabetic patients or on steroids;

- Reviewing of blood glucose management in last days of life;

- Accurate recording and prescribing of blood glucose lowering drugs.

Secondly, recurring multifaceted and multidisciplinary education of staff on diabetes and the importance of blood sugar management, supported by publicity about the new policy and hospice standards in all clinical areas.

Thirdly, change of existing blood glucose monitoring proforma to include more detail and increased guidance regarding target blood glucose levels.

Results Initial audit demonstrated inadequate management plans, no blood glucose target range setting, poor blood glucose monitoring, no last days of life review of management or monitoring of blood glucose levels for patients on steroids.

Second audit demonstrated significant improvement in management of patients with diabetes, including at end of life but continued poor monitoring and management of blood glucose in patients with steroids.

Final audit demonstrated high compliance in all areas.

Conclusion Evolution of clinical management culture may be challenging and prolonged. Combination of techniques is necessary, including repeated, multidisciplinary education of the underlying issues, publicity regarding change, practical guidance for staff and revolution in aspects of multidisciplinary working and care. 